TITLE:

\title{
Monotone Smoothing Splines using General Linear Systems
}

$\operatorname{AUTHOR}(\mathrm{S})$ :

Nagahara, Masaaki; Martin, Clyde F.

CITATION:

Nagahara, Masaaki ...[et al]. Monotone Smoothing Splines using

General Linear Systems. Asian Journal of Control 2013, 15(2): 461-468

ISSUE DATE:

2013-03

URL:

http://hdl.handle.net/2433/173110

\section{RIGHT:}

(c) 2012 John Wiley and Sons Asia Pte Ltd and Chinese Automatic Control Society; The definitive version is available at www3.interscience.wiley.com; This is not the published version. Please cite only the published version.; この論文は出版社版でありません。引用 の際には出版社版をご確認ご利用ください。 


\title{
MONOTONE SMOOTHING SPLINES USING GENERAL LINEAR SYSTEMS
}

\author{
MASAAKI NAGAHARA AND CLYDE F. MARTIN
}

\begin{abstract}
In this paper, a method is proposed to solve the problem of monotone smoothing splines using general linear systems. This problem, also called monotone control theoretic splines, has been solved only when the curve generator is modeled by the second-order integrator, but not for other cases. The difficulty in the problem is that the monotonicity constraint should be satisfied over an interval which has the cardinality of the continuum. To solve this problem, we first formulate the problem as a semi-infinite quadratic programming, and then we adopt a discretization technique to obtain a finitedimensional quadratic programming problem. It is shown that the solution of the finite-dimensional problem always satisfies the infinite-dimensional monotonicity constraint. It is also proved that the approximated solution converges to the exact solution as the discretization grid-size tends to zero. An example is presented to show the effectiveness of the proposed method.
\end{abstract}

\section{INTRODUCTION}

Control theoretic splines are either interpolating or smoothing splines, depending on the constraints and cost function, with a constraint written as a system of linear differential equations [25]. The spline curve is obtained as the output of a given linear system. This concept is generalization of the smoothing spline by Wahba $[17,28]$ in that a richer class of smoothing curves can be obtained relative to polynomial curves. They have been proved to be useful in trajectory planning $[8$, 22], mobile robots [26], contour modeling of images [15], probability distribution estimation [3, 4], and so on. For more applications and a rather complete theory of control theoretic splines, see the book [10].

On the other hand, monotone control theoretic splines are also important in deriving a model or an estimation of a parameter such as the growing rate of an individual $[7,9,10]$. Also, there have been a few applications of monotone smoothing in the statistical literature. The work of Ramsay [21] and the discussion associated with the paper seems to be the first use. A more recent paper by Kelly [16] is a nice application.

Monotonicity is achieved by adding a non-negative (or non-positive) derivative constraint on the output of the target linear system over a time interval. Since the interval is an infinite subset of $[0, \infty)$, this constraint becomes infinite-dimensional, which makes the problem difficult to solve. In [7, 9], this has been solved when the target linear system is a second-order integrator $1 / s^{2}$. However, for general cases,

Key words and phrases. Smoothing splines, optimal control, semi-infinite optimization.

ASIAN JOURNAL OF CONTROL, VOL. 15, NO. 2, PP. 461-468, MARCH 2013.

PUBLISHED ONLINE 25 JUNE 2012 IN WILEY ONLINE LIBRARY (WILEYONLINELIBRARY.COM) DOI: 10.1002/ASJC.557 
no solution has been obtained. More recently, the smoothing problem has been solved with inequality constraints [1], in which inequality constraints are defined only on sampling points, and the constraints may be violated between sampling points.

In this paper, we propose a new method for constructing monotone splines guaranteeing the inequality constraint on a whole time interval, say $[0, T]$, with more general linear systems. To solve this, we first formulate the problem as a semiinfinite quadratic programming problem $[13,19]$. Then we adopt a discretization technique $[14,27]$ to reduce the infinite-dimensional constraints to a finite dimensional constraints. This discretization is executed by partitioning the time interval into finitely many subintervals and estimating the constraint on a finite grid. The finite-dimensional quadratic programming problem can be solved with efficient algorithms [2].

We also discuss the limiting properties of the approximated solution. Whereas the approximated solution is constrained on a finite grid, we show that it satisfies the original infinite-dimensional constraint provided the grid size is sufficiently small. We also prove that the approximated solution converges to the exact solution as the discretization grid-size tends to zero. By these properties, the proposed method can be safely adopted for monotone control theoretic splines. We also construct an illustrative example to show the effectiveness of our method.

The method proposed in this paper can be directly applied to model predictive control (MPC), in particular nonuniformly-sampled-data MPC with continuoustime inequality constraints. For the study, there are many researches; MPC with uniform sampling and piecewise constant control [20,12], with constraints only on the sampling points [6], and with nonuniformly sampling but unconstrained [24]. To our knowledge, there is no method for nonuniformly-sampled-data MPC with relatively general control inputs which guarantees continuous-time constraints as we will consider in this paper.

The organization of this paper is as follows: Section 2 defines the problem of monotone control theoretic splines. In Section 3, we formulate the problem as a semi-infinite quadratic programming. Section 4 is the main section, where discretization approach is introduced and the limiting properties are discussed. Section 5 suggests a formula for computing inner products used in our optimization. A numerical example is illustrated in Section 6, and a conclusion is made in Section 7.

Notation. In this paper, we use the following notation. $\mathbb{R}, \mathbb{R}^{n}$, and $\mathbb{R}^{n \times n}$ are respectively the set of real numbers, $n$-dimensional real vectors and $n \times n$ matrices. We denote $L^{2}[0, T]$ by the Lebesgue space consisting of all square integrable real functions on $[0, T] \subset \mathbb{R}$, endowed with the inner product

$$
\langle x, y\rangle:=\int_{0}^{T} x(t) y(t) \mathrm{d} t, \quad x, y \in L^{2}[0, T] .
$$

For a matrix (a vector) $M, M^{\top}$ is the transpose of $M$. For a vector $v=\left[v_{1}, \ldots, v_{n}\right]^{\top} \in$ $\mathbb{R}^{n}, v \preceq 0$ means $v_{i} \leq 0$ for $i=1,2, \ldots, n$, and for two vectors $v$ and $w, v \preceq w$ if $v-w \preceq 0$. For a vector $v \in \mathbb{R}^{n}$,

$$
\begin{aligned}
\|v\|_{1} & :=\sum_{i=1}^{n}\left|v_{i}\right|, \\
\|v\|_{\infty} & :=\max _{i=1, \ldots, n}\left|v_{i}\right| .
\end{aligned}
$$




\section{Monotone Smoothing Splines}

In this paper, we consider the following problem of monotone smoothing splines:

Problem 1 (Monotone smoothing splines). Given a linear time-invariant system

$$
\dot{x}=A x+B u, \quad y=C x, x(0)=x_{0} \in \mathbb{R}^{n},
$$

where $x(t) \in \mathbb{R}^{n}$ is the state vector, $u(t) \in \mathbb{R}$ is the control input, $y(t) \in \mathbb{R}$ is the plant output, $A \in \mathbb{R}^{n \times n}, B \in \mathbb{R}^{n \times 1}, C \in \mathbb{R}^{1 \times n}$, and also given a data set

$$
\mathcal{D}:=\left\{\left(t_{1}, \alpha_{1}\right),\left(t_{2}, \alpha_{2}\right), \ldots,\left(t_{m}, \alpha_{m}\right)\right\},
$$

with time instants $0<t_{1}<t_{2}<\cdots<t_{m}=: T$ and noisy data $\alpha_{1}, \ldots, \alpha_{m} \in \mathbb{R}$ on the time instants, find the control $u \in L^{2}[0, T]$ and the initial condition $x(0)=$ $x_{0} \in \mathbb{R}^{n}$ that minimizes the following cost function:

$$
J(u):=\lambda \int_{0}^{T} u(t)^{2} \mathrm{~d} t+\sum_{i=1}^{m} w_{i}\left(y\left(t_{i}\right)-\alpha_{i}\right)^{2},
$$

where $\lambda$ and $w_{i}$ 's are given positive numbers (weights), subject to the monotonicity constraint

$$
\dot{y}(t) \geq 0, \quad \forall t \in[0, T] .
$$

The cost function (1) considers elimination of Gaussian noise on the data $\left\{\alpha_{1}, \ldots, \alpha_{m}\right\}$ and limitation on the $L^{2}$ norm of the control $u$. This formulation is an extension of Wahba's smoothing spline [17, 28].

This problem has been solved in $[7,9]$ only when $C(s I-A)^{-1} B=1 / s^{2}$. However, for other cases, the problem has not been solved.

Remark 1. One might think that the problem can be solved via a technique of model predictive control $(M P C)$. The difficulty in using $M P C$ is that we have to treat the constraint (2) which is infinite dimensional. If we discretize this constraint as $\dot{y}\left(t_{i}\right) \geq 0 i=1,2, \ldots, m$ to use a standard MPC formulation, the original constraint (2) will be guaranteed only on the sampling points $t_{1}, \ldots, t_{m}$, not on the whole $[0, T]$. As mentioned in Section 1, there is no method for the problem given in (1) and (2) within the framework of $M P C$.

\section{Formulation by Semi-Infinite Quadratic Programming}

We here formulate the problem given in the previous section by semi-infinite quadratic programming. We first assume that we search the optimal control $u$ in the following subspace of $L^{2}[0, T]$ as taken in [25]:

$$
V_{m}:=\left\{u \in L^{2}[0, T]: u=\sum_{i=1}^{m} \eta_{i} \phi_{t_{i}}, \eta_{i} \in \mathbb{R}\right\},
$$

where $\phi_{t} \in L^{2}[0, T]$ is defined by

$$
\phi_{t}(\tau):= \begin{cases}C e^{A(t-\tau)} B, & t>\tau, \\ 0, & t \leq \tau .\end{cases}
$$

This characterization is based on the representer theorem $[17,28,23]$ for the regularized cost functional (1) with the kernel $k(t, \tau)=\left\langle\phi_{t}, \phi_{\tau}\right\rangle$; the optimal estimation 
$y(t)$ is represented by

$$
y(t)=\sum_{i=1}^{m} \eta_{i} k\left(t, t_{i}\right)=\sum_{i=1}^{m} \eta_{i}\left\langle\phi_{t}, \phi_{t_{i}}\right\rangle .
$$

One can consider other base functions $\left\{\phi_{i}\right\}_{i=1}^{M}$ than (3) and define the subspace $V_{M}:=\operatorname{span}\left\{\phi_{i}\right\}_{i=1}^{M}$ where $M \gg m$. This is related to basis pursuit denoising [5], with which we are not concerned in this paper.

By assuming that the control $u$ is in the subspace $V_{m}$, the cost function (1) can be described as [25]

$$
J\left(\sum_{i=1}^{m} \eta_{i} \phi_{t_{i}}\right)=\frac{1}{2} \theta^{\top} P \theta+q^{\top} \theta+\alpha^{\top} W \alpha,
$$

where $\theta:=\left[\eta_{1}, \ldots, \eta_{m}, x_{0}^{\top}\right]^{\top}, \alpha:=\left[\alpha_{1}, \ldots, \alpha_{m}\right]^{\top}$, and

$$
\begin{aligned}
P & :=\left[\begin{array}{ccc}
2(\lambda I+G W) G & 2 G W F \\
2 F^{\top} W G & 2 F^{\top} W F
\end{array}\right], \quad q:=-2[G F]^{\top} W \alpha, \\
G & :=\left[\begin{array}{cccc}
\left\langle\phi_{t_{1}}, \phi_{t_{1}}\right\rangle & \left\langle\phi_{t_{2}}, \phi_{t_{1}}\right\rangle & \ldots & \left\langle\phi_{t_{m}}, \phi_{t_{1}}\right\rangle \\
\vdots & \vdots & \ddots & \vdots \\
\left\langle\phi_{t_{1}}, \phi_{t_{m}}\right\rangle & \left\langle\phi_{t_{2}}, \phi_{t_{m}}\right\rangle & \ldots & \left\langle\phi_{t_{m}}, \phi_{t_{m}}\right\rangle
\end{array}\right], \quad F:=\left[\begin{array}{c}
C e^{A t_{1}} \\
\vdots \\
C e^{A t_{m}}
\end{array}\right], \\
W & :=\operatorname{diag}\left\{w_{1}, \ldots, w_{m}\right\} .
\end{aligned}
$$

Let $\dot{\phi}_{t}(\tau)$ be the derivative of $\phi_{t}(\tau)$, that is,

$$
\dot{\phi}_{t}(\tau):=\frac{\partial \phi_{t}(\tau)}{\partial t}=\left\{\begin{array}{l}
C A e^{A(t-\tau)} B, \quad \text { if } t>\tau, \\
0, \quad \text { if } t \leq \tau .
\end{array}\right.
$$

By using this, the derivative $\dot{y}(t)$ can be calculated as $\dot{y}(t)=\Phi(t)^{\top} \theta$, where

$$
\Phi(t):=\left[\begin{array}{c}
\left\langle\dot{\phi}_{t}, \phi_{t_{1}}\right\rangle \\
\vdots \\
\left\langle\dot{\phi}_{t}, \phi_{t_{m}}\right\rangle \\
e^{A^{\top} t} A^{\top} C^{\top}
\end{array}\right]+C B\left[\begin{array}{c}
\phi_{t_{1}}(t) \\
\vdots \\
\phi_{t_{m}}(t) \\
0
\end{array}\right] .
$$

The constraint (2) now becomes $\Phi(t)^{\top} \theta \geq 0$ for all $t \in[0, T]$. In summary, Problem 1 is formulated by the following:

Problem 2. Find $\theta \in \mathbb{R}^{m+n}$ that minimizes

$$
f(\theta)=\frac{1}{2} \theta^{\top} P \theta+q^{\top} \theta
$$

subject to

$$
g(\theta, t)=-\Phi(t)^{\top} \theta \leq 0, \quad \text { for all } t \in[0, T] .
$$

Note that the term $\alpha^{\top} W \alpha$ in (4) is omitted in (6) since this term is constant (independent of $\theta$ ) and does not change the optimal solution. Problem 2 is a semi-infinite quadratic programming problem. By the optimal solution $\theta^{*}$ to this problem, we obtain the optimal control input $u^{*}$ by

$$
u^{*}(t)=\sum_{i=1}^{m} \theta^{*}[i] \phi_{t_{i}}(t)
$$

where $\theta^{*}[i]$ is the $i$-th element of $\theta^{*}$. 


\section{Discretization Approach to Monotone Splines}

4.1. Discretization. The difficulty in Problem 2 is that the inequality constraint (7) is infinite dimensional. We here introduce an approximation technique for such an infinite-dimensional constraint. To reduce this to a finite dimensional one, we adopt the technique of discretization [14, 27].

We first divide the interval $[0, T]$ into $M$ subintervals: $[0, T]=\left[T_{0}, T_{1}\right] \cup \cdots \cup$ $\left(T_{M-1}, T_{M}\right]$, where $\left\{T_{i}\right\}$ is the discretization grid satisfying $0=T_{0}<T_{1}<\cdots<$ $T_{M}=T$. Then we evaluate the function $g(\theta, t)$ in $(7)$ on the grid points $t=$ $T_{0}, T_{1}, \ldots, T_{M}$. To guarantee the constraint (7), we choose real numbers $\epsilon>0$ and $r>0$ and adopt the following constraints:

$$
g\left(\theta, T_{0}\right) \leq-\epsilon, \ldots, g\left(\theta, T_{M}\right) \leq-\epsilon,-r \mathbf{1} \preceq \theta \preceq r \mathbf{1}
$$

where $\mathbf{1}=[1,1, \ldots, 1]^{\top} \in \mathbb{R}^{m+n}$. The second inequality is component wise (see Notation in Section 1), and means $\|\theta\|_{\infty} \leq r$. The finite-dimensional constraints (8) can be represented as a matrix inequality,

$$
H \theta \preceq v,
$$

where

$$
\begin{aligned}
H & :=\left[\begin{array}{lllll}
\Phi\left(T_{0}\right) & \ldots & \Phi\left(T_{M}\right) & I & -I
\end{array}\right]^{\top}, \\
v & :=\left[\begin{array}{lllll}
-\epsilon & \ldots & -\epsilon & r \mathbf{1}^{\top} & r \mathbf{1}^{\top}
\end{array}\right]^{\top} .
\end{aligned}
$$

In summary, the problem of monotone control theoretic splines for arbitrarily linear time-invariant system $\{A, B, C\}$ can be approximately described by the following quadratic programming: find $\theta \in \mathbb{R}^{m+n}$ which minimizes (6) subject to (9). This is a standard quadratic programming and can be efficiently solved by numerical softwares such as MATLAB.

Remark 2. We can also include equality constraints into our optimization. For example, if we want to have $y(T)=1$ and $\dot{y}(T)=0$, then our constraints are represented by

$$
\begin{aligned}
{\left[\left\langle\phi_{T}, \phi_{t_{1}}\right\rangle, \ldots,\left\langle\phi_{T}, \phi_{t_{m}}\right\rangle, C e^{A T}\right] \theta } & =1, \\
{\left[\left\langle\dot{\phi}_{T}, \phi_{t_{1}}\right\rangle, \ldots,\left\langle\dot{\phi}_{T}, \phi_{t_{m}}\right\rangle, C A e^{A T}\right] \theta } & =0 .
\end{aligned}
$$

These constraints are finite dimensional and can be included easily in our quadratic programming. In general, equality constraints on the output $y$ and the derivative $\dot{y}$ on time instants in $[0, T]$ can be represented by linear constraints as above.

Remark 3. Splines with another constraint than monotonicity such as concavity, i.e., $\ddot{y}(t) \leq 0$, can be also solved by the same method.

4.2. Convergence analysis. Put $N:=m+n$. Define feasible sets $\mathcal{F}$ for the original optimization and $\widetilde{\mathcal{F}}(M, \epsilon, r)$ for the approximation respectively by

$$
\begin{gathered}
\mathcal{F}:=\left\{\theta \in \mathbb{R}^{N}: g(\theta, t) \leq 0, t \in[0, T]\right\}, \\
\widetilde{\mathcal{F}}(M, \epsilon, r):=\left\{\theta \in \mathbb{R}^{N}: g(\theta, t) \leq-\epsilon, t \in\left\{T_{0}, \ldots, T_{M}\right\},\|\theta\|_{\infty} \leq r\right\} .
\end{gathered}
$$

To prove our first proposition, we assume the following:

Assumption 1. The function $\Phi(t)$ is Lipschitz continuous, that is, there exists a real number $\mu>0$ such that $\|\Phi(t)-\Phi(s)\|_{1} \leq \mu|t-s|$ for all $t, s \in[0, T]$. 
Remark 4. Systems whose relative degree is higher than or equal to 2 satisfy the above assumption. First, by the definition of $\dot{\phi}_{t}(\tau)$ in (5), we can say that for $j=1,2, \ldots, m, \frac{d}{d t}\left\langle\dot{\phi}_{t}, \phi_{t_{j}}\right\rangle$ is bounded on $[0, T]$, and hence $\left\langle\dot{\phi}_{t}, \phi_{t_{j}}\right\rangle$ is Lipschitz on $[0, T]$. Also, the function $e^{A^{\top} t} A^{\top} C^{\top}$ is Lipschitz. These facts and $C B=0$ (since the relative degree $\geq 2$ ) result in that $\Phi(t)$ is Lipschitz.

Define the discretization grid-size by

$$
I_{\max }(M):=\max \left\{\left|T_{i}-T_{i-1}\right|: i=1,2, \ldots, M\right\} .
$$

We then have the following proposition:

Proposition 1. Suppose that Assumption 1 is satisfied. Suppose also that $M, \epsilon$ and $r$ are chosen such that

$$
I_{\max }(M) \leq \frac{\epsilon}{r \mu} .
$$

Then we have $\widetilde{\mathcal{F}}(M, \epsilon, r) \subseteq \mathcal{F}$.

Proof. Let $\theta \in \widetilde{\mathcal{F}}(M, \epsilon, r)$. By the definition of $\widetilde{\mathcal{F}}(M, \epsilon, r)$ in (11), $\theta$ satisfies $g\left(\theta, T_{i}\right) \leq-\epsilon, i=0,1, \ldots, M$. Then, for any $t \in[0, T]$, there exists $i \in\{1, \ldots, M\}$ such that $t \in\left[T_{i-1}, T_{i}\right]$. By Assumption 1 and the condition $\|\theta\|_{\infty} \leq r$ in (11), we have

$$
g(\theta, t)-g\left(\theta, T_{i}\right)=-\left(\Phi(t)-\Phi\left(T_{i}\right)\right)^{\top} \theta \leq r \mu\left(T_{i}-t\right) .
$$

By this inequality, we have

$$
g(\theta, t) \leq r \mu\left(T_{i}-t\right)+g\left(\theta, T_{i}\right) \leq r \mu I_{\max }(M)-\epsilon \leq 0 .
$$

The last inequality is due to (12). Thus, $\theta \in \mathcal{F}$ and hence $\widetilde{\mathcal{F}}(M, \epsilon, r) \subseteq \mathcal{F}$.

By this proposition, we can guarantee the constraint (7) by searching the parameter $\theta$ in the finite-dimensional feasible set $\widetilde{\mathcal{F}}(M, \epsilon, r)$ provided that the number $M$ is sufficiently large to satisfy (12).

Next, we analyze the limiting property of the approximated solution when the discretization grid size tends to zero. To prove the property, we assume the following:

Assumption 2. The interior of $\mathcal{F}$

$$
\text { int } \mathcal{F}:=\left\{\theta \in \mathbb{R}^{N}: g(\theta, t)<0 \text {, for all } t \in[0, T]\right\}
$$

is nonempty.

Remark 5. Assumption 2 is slightly restrictive. A sufficient condition for this is that $A$ is a Metzler matrix ${ }^{1}$ and $C A \succeq 0(C A \not \equiv 0)$. By the positive system theory [11], $A$ is Metzler if and only if $e^{A t} \succeq 0$ for all $t \in \mathbb{R}$. Let $u \equiv 0$. Then, we have $\dot{y}(t)=C A e^{A t} x_{0} \geq 0$ for all $t \in \mathbb{R}$. Since $C A e^{A t} x_{0}$ is continuous on $\mathbb{R}$, we have $\dot{y}(t)=C A e^{A t} x_{0}>0$ for all $t \in[0, T]$, if we choose sufficient large $x_{0} \succ 0$. Therefore, int $\mathcal{F}$ is nonempty in this case.

Then we have the following theorem:

Theorem 1. Suppose that Assumptions 1 and 2 are satisfied. Let $M(\epsilon, r)$ be the minimum value among all $M$ 's satisfying (12). For $f(\theta)$ given in (6), define

$$
\theta^{*}:=\underset{\theta \in \mathcal{F}}{\arg \min } f(\theta), \quad \theta_{\epsilon, r}^{*}:=\underset{\theta \in \widetilde{\mathcal{F}}(M(\epsilon, r), \epsilon, r)}{\arg \min } f(\theta) .
$$

\footnotetext{
${ }^{1}$ A matrix $A$ is called a Metzler matrix if all the off-diagonal components are nonnegative.
} 
Then we have $f\left(\theta_{\epsilon, r}^{*}\right) \rightarrow f\left(\theta^{*}\right)$ as $\epsilon \rightarrow+0$ and $r \rightarrow \infty$.

Proof. Since int $\mathcal{F}$ is nonempty, there exist $\bar{\theta} \in \operatorname{int} \mathcal{F}$. It follows that there exists $\delta>0$ such that

$$
g(\bar{\theta}, t) \leq-\delta, \quad \forall t \in[0, T] .
$$

Then, since $\mathcal{F}$ is a convex set, we have $\theta_{\xi}:=\xi \bar{\theta}+(1-\xi) \theta^{*} \in \operatorname{int} \mathcal{F}$ for all $\xi \in(0,1]$. Fix arbitrarily $\xi \in(0,1]$. Since $f$ is a convex function, we have

$$
f\left(\theta_{\xi}\right) \leq \xi(\bar{\theta})+(1-\xi) f\left(\theta^{*}\right)=f\left(\theta^{*}\right)+\xi \Delta,
$$

where $\Delta:=f(\bar{\theta})-f\left(\theta^{*}\right)$. Note that $\Delta \geq 0$ by the definition of $\theta^{*}$. Also, for all $t \in[0, T]$, we have

$$
g\left(\theta_{\xi}, t\right)=-\Phi(t)^{\top} \theta_{\xi}=\xi g(\bar{\theta}, t)+(1-\xi) g\left(\theta^{*}, t\right) \leq-\xi \delta,
$$

where the last inequality is due to inequality (13) and $g\left(\theta^{*}, t\right) \leq 0$ for $\theta^{*} \in \mathcal{F}$ defined in (10). Let $\bar{r}:=\max _{\xi \in(0,1]}\left\|\theta_{\xi}\right\|_{\infty}$ and fix arbitrarily $r \geq \bar{r}$. Then we have $\theta_{\xi} \in \widetilde{\mathcal{F}}(M(\xi \delta, r), \xi \delta, r)$. This gives the following inequality:

$$
f\left(\theta_{\xi \delta, r}^{*}\right) \leq f\left(\theta_{\xi}\right) \leq f\left(\theta^{*}\right)+\xi \Delta .
$$

On the other hand, by Proposition 1 , we have $\mathcal{F} \supseteq \widetilde{\mathcal{F}}(M(\xi \delta, r), \xi \delta, r)$, and hence

$$
f\left(\theta^{*}\right) \leq f\left(\theta_{\xi \delta, r}^{*}\right) .
$$

Combining (15) and (16) gives $f\left(\theta^{*}\right) \leq f\left(\theta_{\xi \delta, r}^{*}\right) \leq f\left(\theta^{*}\right)+\xi \Delta$. We thus have

$$
\lim _{\substack{\epsilon \rightarrow+0 \\ r \rightarrow \infty}} f\left(\theta_{\epsilon, r}^{*}\right)=\lim _{\substack{\xi \rightarrow+0 \\ r \rightarrow \infty}} f\left(\theta_{\xi \delta, r}^{*}\right)=f\left(\theta^{*}\right) .
$$

Remark 6. By Theorem 1, the semi-infinite programming we consider here is weakly discretizable [19]. Note that this does not necessarily imply that $\theta_{\epsilon, r}^{*} \rightarrow \theta^{*}$.

By Theorem 1, the proposed approximation method given in the previous section can be safely adopted for monotone control theoretic splines.

\section{Computing Inner Product}

To compute the Grammian $G$ and the matrix $\Phi(t)$, we have to compute the following inner products for fixed $s, t \in[0, T]$ :

$$
\begin{aligned}
& \left\langle\phi_{s}, \phi_{t}\right\rangle=\int_{0}^{T} \phi_{s}(\tau) \phi_{t}(\tau) \mathrm{d} \tau, \\
& \left\langle\dot{\phi}_{s}, \phi_{t}\right\rangle=\int_{0}^{T} \dot{\phi}_{s}(\tau) \phi_{t}(\tau) \mathrm{d} \tau .
\end{aligned}
$$

These values can be easily computed by matrix exponentials [18]:

$$
\begin{aligned}
\left\langle\phi_{s}, \phi_{t}\right\rangle & =B^{\top} e^{A^{\top} s}\left(\int_{0}^{h} e^{-A^{\top} \tau} C^{\top} C e^{-A \tau} \mathrm{d} \tau\right) e^{A t} B \\
& =: v_{s}^{\top}\left(F_{22}^{\top} F_{12}\right) v_{t},
\end{aligned}
$$

where $h:=\min (s, t), v_{\tau}:=e^{A \tau} B(\tau=t, s)$, and the matrices $F_{22}$ and $F_{12}$ are defined by

$$
\left[\begin{array}{cc}
F_{11} & F_{12} \\
0 & F_{22}
\end{array}\right]:=\exp \left(\left[\begin{array}{cc}
A^{\top} & C^{\top} C \\
0 & -A
\end{array}\right] h\right) \text {. }
$$




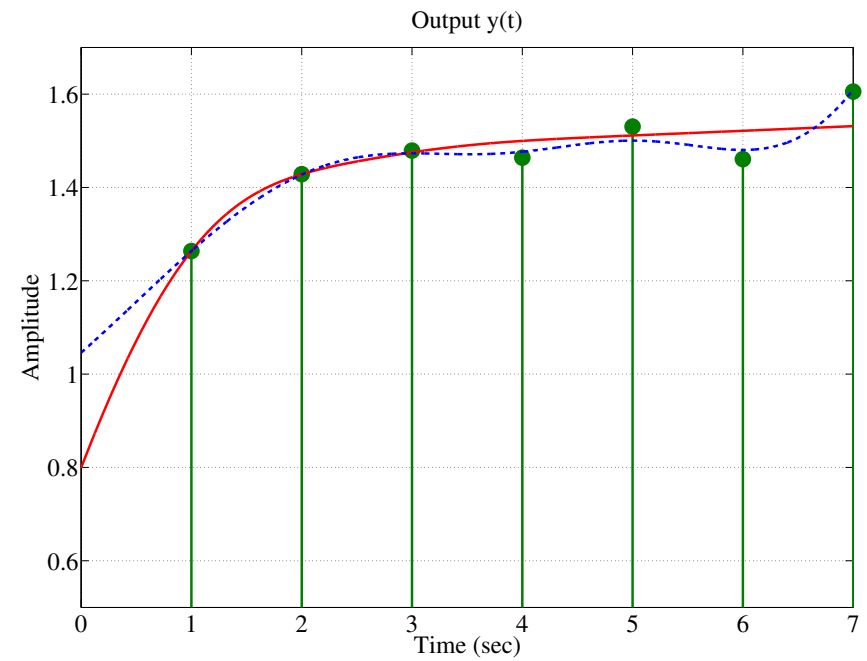

Figure 1. Data (circles) and estimation $y(t)$ by the proposed method (solid), and a conventional discretization method (dash)

The inner product $\left\langle\dot{\phi}_{s}, \phi_{t}\right\rangle$ is also obtained by

$$
\begin{aligned}
\left\langle\dot{\phi}_{s}, \phi_{t}\right\rangle & =v_{s}^{\top}\left(H_{22}^{\top} H_{12}\right) v_{t} \\
{\left[\begin{array}{cc}
H_{11} & H_{12} \\
0 & H_{22}
\end{array}\right] } & :=\exp \left(\left[\begin{array}{cc}
A^{\top} & A^{\top} C^{\top} C \\
0 & -A
\end{array}\right] h\right) .
\end{aligned}
$$

\section{EXAMPLE}

We here show an example of monotone control theoretic splines. Assume that the system is given by

$$
P(s)=\frac{1}{s^{2}\left(s^{2}+1\right)} .
$$

Note that for the second order integrator $1 / s^{2}$ the exact optimal solution can be obtained [7,9], but for higher order systems as in (17), there has been no method to construct monotone smoothing splines. Let the original curve be $y_{\text {orig }}(t)=1.5-e^{-t}$, and the noisy data is obtained by

$$
t_{i}=i, \quad \alpha_{i}=y_{\text {orig }}\left(t_{i}\right)+\varepsilon_{i}, \quad i=1, \ldots, 7,
$$

where $\varepsilon_{i}$ is an independently and identically distributed random variable of the Gaussian distribution $\mathcal{N}(0,0.05)$. The estimation result is shown in Fig. 1. We also calculate the estimation by a standard method as used in [1] with the monotone constraint only on the points $t=0,1, \ldots, 7$. This figure shows that our estimation works well while the conventional one shows oscilations in the intervals $(3,4)$ and $(5,6)$, which indicates that the derivative is negative. To see this more precisely, we show the derivative $\dot{y}(t)$ in Fig. 2. We can see that the derivative by the conventional method is non-negative on the sampling points $t=0,1, \ldots, 7$, however the constraint is violated in the intervals $(3,4)$ and $(5,6)$ (See Fig. 3 for an enlarged plot). On the other hand, the derivative by our method is always non-negative on the whole interval $t \in[0,7]$. Fig. 4 shows the input $u(t)$ for the both methods. By 


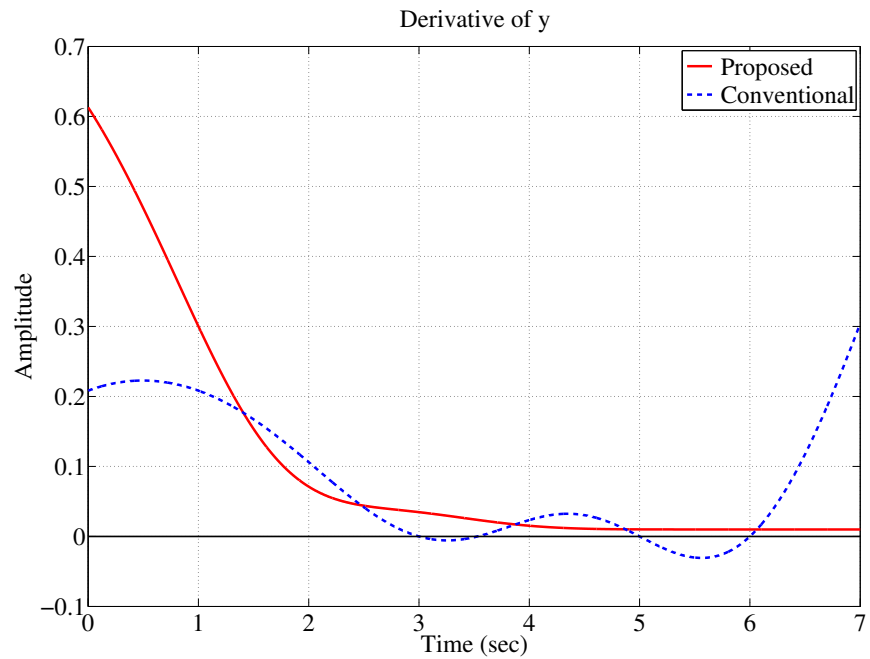

Figure 2. Derivative of estimation $y(t)$ by the proposed method (solid), and a conventional discretization method (dash)

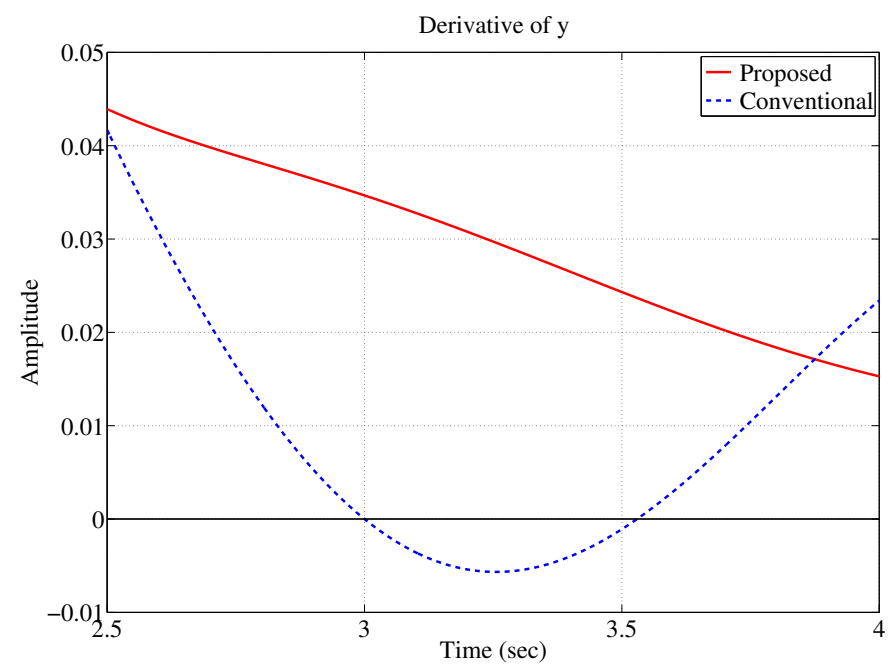

Figure 3. Enlarged plot of Fig. 2

this figure, we can see that the input signal $u(t)$ of the conventional method shows large gain on the intervals $(3,4)$ and $(5,6)$ where the conventional method violates the monotonicity constraint.

\section{Conclusion}

In this paper, we have proposed a new method for solving the problem of monotone control theoretic splines for general linear systems. By using discretization technique, the problem is approximately described as a finite-dimensional quadratic 


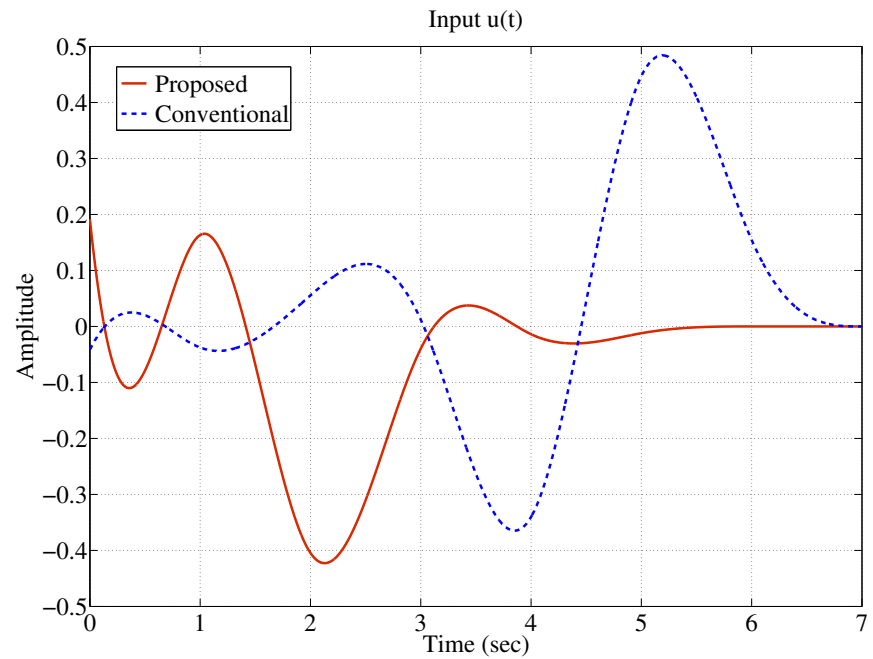

FiguRE 4. Input $u(t)$ : proposed (solid) and conventional (dash)

programming. The optimal parameters can be efficiently obtained by numerical optimization softwares such as MATLAB. The obtained estimation is guaranteed to satisfy the monotonicity constraint. We also proved limiting properties of approximated solutions. Future work may include quantification of the error introduced by the proposed approximation, and also construction of monotone control theoretic splines for MIMO (multi-input multi-output) systems.

\section{ACKNOWLEDGEMENT}

This research was supported in part by Grand-in-Aid for Young Scientists (B) of Japan Society for the Promotion of Science under Grant No. 22760317.

\section{REFERENCES}

[1] Bell B. M., J. V. Burke, and G. Pillonetto, "An inequality constrained nonlinear Kalman-Bucy smoother by interior point likelihood maximization," Automatica, Vol. 45, No. 1, pp. 25-33 (2009).

[2] Boyd S. and L. Vandenberghe, Convex Optimization, Cambridge (2004).

[3] Charles J. K., Probability Distribution Estimation Using Control Theoretic Splines, Doctoral Dissertation, Texas Tech University (2009).

[4] Charles J. K., S. Sun, and C. F. Martin, "Cumulative distribution estimation via control theoretic smoothing splines," Three Decades of Progress in Control Sciences, Springer, pp. 83-92 (2010).

[5] Chen S. S., D. L. Donoho, and M. A. Saunders, "Atomic decomposition by basis pursuit," SIAM Journal on Scientific Computing, Vol. 20, No. 1, pp. 33-61 (1998).

[6] Diehl M., H. G. Bock, J. P. Schlöder, R. Findeisen, Z. Nagy, and F. Allgöwer, "Real-time optimization and nonlinear model predictive control of processes governed by differentialalgebraic equations," Journal of Process Control, Vol. 12, No. 4, pp. 577-585 (2002).

[7] Egerstedt M. and C. F. Martin, "Monotone smoothing splines," Proceedings of the Mathematical Theory of Networks and Systems, pp. 1020-1028 (2000).

[8] Egerstedt M. and C. F. Martin, "Optimal trajectory planning and smoothing splines," $A u$ tomatica, Vol. 37, No. 7, pp. 1057-1064 (2001). 
[9] Egerstedt M. and C. F. Martin, "Optimal control and monotone smoothing splines," New Trends in Nonlinear Dynamics and Control, Springer, pp. 279-294 (2003).

[10] Egerstedt M. and C. F. Martin, Control Theoretic Splines, Princeton University Press (2010).

[11] Farina L. and S. Rinaldi, Positive Linear Systems: Theory and Applications, WileyInterscience (2000).

[12] Grüne L., D. Nešić, and J. Pannek, "Model predictive control for nonlinear sampled-data systems," Assessment and Future Directions of Nonlinear Model Predictive Control, Springer, pp. 105-113 (2007).

[13] Hettich R. and K. O. Kortanek, "Semi-infinite programming: theory, methods, and applications," SIAM Review, Vol. 35, No. 3, pp. 380-429 (1993).

[14] Hettich R. and G. Gramlich, "A note on an implementation of a method for quadratic semiinfinite programming," Mathematical Programming, Vol. 46, No. 2, pp. 249-254 (1990).

[15] Kano H., M. Egerstedt, H. Fujioka, S. Takahashi, and C. F. Martin, "Periodic smoothing splines," Automatica, Vol. 44, No. 1, pp. 185-192 (2008).

[16] Kelly C. and J. Rice, "Monotone smoothing with application to dose-response curves and the assessment of synergism," Biometrics, Vol. 46, pp. 1071-1085 (1990).

[17] Kimeldorf G. and G. Wahba, "Some results on Tchebycheffian spline functions," Journal of Mathematical Analysis and Applications Vol. 33, No. 1, pp. $82-95$ (1971).

[18] Loan C. F. V., "Computing integrals involving the matrix exponential," IEEE Trans. on Automatic Control, Vol. 23, No. 3, pp. 395-404 (1978).

[19] López M. and G. Still, "Semi-infinite programming," European Journal of Operational Research, Vol. 180, No. 2, pp. 491-518 (2007).

[20] Magni L. and R. Scattolini, "Model predictive control of continuous-time nonlinear systems with piecewise constant control," IEEE Trans. Automatic Control, pp. 49, No. 6, pp. 900-906 (2004).

[21] Ramsay J. O., "Monotone regression splines in action," Statistical Science, Vol. 3, pp. 425-461 (1988).

[22] F. J. Rubio, F. J. Valero, J. L. Suñer, and V. Mata, "Simultaneous algorithm for trajectory planning," Asian Journal of Control, Vol. 12, No. 4, pp. 468-479 (2010).

[23] Schölkopf B. and A. J. Smola, Learning with Kernels, The MIT Press (2002).

[24] Sheng J., T. Chen, and S. L. Shah, "Generalized predictive control for non-uniformly sampled systems," Journal of Process Control, Vol. 12, No. 8, pp. 875-885 (2002).

[25] Sun S., M. B. Egerstedt, and C. F. Martin, "Control theoretic smoothing splines," IEEE Trans. on Automatic Control Vol. 45, No 12, pp. 2271-2279 (2000).

[26] Takahashi S. and C. F. Martin, "Optimal control theoretic splines and its application to mobile robot," Proc. of the 2004 IEEE CCA, pp. 1729-1732 (2004).

[27] Teo K. L. and X. Q. Yang, "Computational discretization algorithms for functional inequality constrained optimization," Annals of Operations Research, Vol. 98, pp. 215-234 (2000).

[28] Wahba G., Spline Models for Observational Data, SIAM (1990).

M. Nagahara is with Graduate School of Informatics, Kyoto University, Kyoto 6068501, Japan. (nagahara@ieee.org). C. F. Martin is with Department of Mathematics \& Statistics, Texas Tech University, Texas, USA.(clyde.f.martin@ttu.edu). M. Nagahara IS THE CORRESPONDING AUTHOR. 